\title{
THE RANGE OF A VECTOR MEASURE
}

\author{
PAUL R. HALMOS
}

The purpose of this note is to prove that the range of a countably additive finite measure with values in a finite-dimensional real vector space is closed and, in the non-atomic case, convex. These results were first proved (in 1940) by A. Liapounoff. ${ }^{1}$ In 1945 K. R. Buch (independently) proved the part of the statement concerning closure for non-negative measures of dimension one or two. ${ }^{2}$ In 1947 I offered a proof of Buch's results which, however, was correct in the onedimensional case only. ${ }^{3}$ In this paper I present a simplified proof of Liapounoff's results.

Let $X$ be any set and let $S$ be a $\sigma$-field of subsets of $X$ (called the measurable set of $X$ ). A measure $\mu$ (or, more precisely, an $N$-dimensional measure $\left.\left(\mu_{1}, \cdots, \mu_{N}\right)\right)$ is a (bounded) countably additive function of the sets of $S$ with values in $N$-dimensional, real vector space (in which the "length" $\left|\xi_{1}\right|+\cdots+\left|\xi_{N}\right|$ of a vector $\xi=\left(\xi_{1}, \cdots, \xi_{N}\right)$ is denoted by $\left.|\xi|\right)$. The measure $\left(\mu_{1}, \cdots \mu_{N}\right)$ is non-negative if $\mu_{i}(E) \geqq 0$ for every $E \in S$ and $i=1, \cdots, N$.

For a numerical (one-dimensional) measure $\mu_{0}, \mu_{0}^{*}(E)$ will denote the total variation of $\mu_{0}$ on $E$; in general, if $\mu=\left(\mu_{1}, \cdots, \mu_{N}\right), \mu^{*}$ will denote the non-negative measure $\left(\mu_{1}^{*}, \cdots, \mu_{N}^{*}\right)$. The length $\left|\mu^{*}\right|$ $=\mu_{1}^{*}+\cdots+\mu_{N}^{*}$ is always a non-negative numerical measure. ${ }^{4}$

Presented to the Society, September 3, 1947; received by the editors July 10, 1947.

1 Sur les fonctions-vecteurs completement additives, Bull. Acad. Sci. URSS. Ser. Math. vol. 4 (1940) pp. 465-478. In a subsequent paper with the same title, in the same journal (vol. 10 (1946) pp. 277-279), Liapounoff gave a very elegant example to show that neither convexity nor closure can always be asserted in the infinite-dimensional case. Related questions for finitely additive measures have been discussed by A. Sobczyk and P. C. Hammer, The ranges of additive set functions, Duke Math. J. vol. 11 (1944) pp. 847-851.

2 Some investigations of the set of values of measures in abstract space. $\mathrm{K}$. Danske Videnskabernes Selskab, Mathematish-Fysishe Meddelelser vol. 21.

- On the set of values of a finite measure, Bull. Amer. Math. Soc. vol. 53 (1947) pp. 138-141. I am indebted to Professors Børge Jessen and Jean Dieudonne for calling my attention to the fact that the statement and proof of Lemma 5 of that paper are wrong. The error in the proof is the implicit assumption that if $S$ is covered by sets of the form $U \cap V$ then the intersection of any $U$ that occurs with any $V$ that occurs is also a set of the covering. It is not difficult to show that the conclusion of Lemma 5 holds if and only if every closed set of either of the two given topologies is compact with respect to the other. It follows that if, for instance, the given topologies are Hausdorff then the conclusion holds if and only if they are identical.

- For the notion of total variation, as well as all other concepts and results of elementary measure theory, see S. Saks, Theory of the integral, Warsaw, 1937, chaps. 1 and 2. 
A measurable set $E$ is an atom of $\mu$ if $\mu(E) \neq 0$ and if for every measurable set $F \subset E$ either $\mu(F)=0$ or $\mu(F)=\mu(E)$. The measure $\mu$ is purely atomic if there exists a sequence $\left\{E_{n}\right\}$ of pairwise disjoint measurable sets such that $X=U_{n} E_{n}$ and every $E_{n}$ is an atom of each $\mu_{i}, i=1, \cdots, N ; \mu$ is purely non-atomic if none of its coordinates $\mu_{i}(i=1, \cdots, N)$ has any atoms.

The measure $\mu$ is absolutely continuous with respect to the measure $\nu$ if $\left|\mu^{*}(E)\right|=0$ for every measurable set $E$ for which $\left|\nu^{*}(E)\right|=0$ (that is, if the numerical measure $\left|\mu^{*}\right|$ is absolutely continuous with respect to the numerical measure $\left.\left|\nu^{*}\right|\right)$. A necessary and sufficient condition that $\mu$ be absolute continuous with respect to $\nu$ is that for every $\epsilon>0$ there exist a $\delta>0$ such that $\left|\mu^{*}(E)\right|<\epsilon$ for every measurable set $E$ for which $\left|\nu^{*}(E)\right|<\delta$. For any two measures $\mu$ and $\nu$ there exists a measurable set $E_{0}$ such that $\left|\nu^{*}\left(E_{0}\right)\right|=0$ and $\left|\mu^{*}\left(E-E_{0}\right)\right|=0$ for every measurable set $E$ for which $\left|\nu^{*}(E)\right|=0$, that is, the measure $\mu^{\prime}$, defined by $\mu^{\prime}(E)=\mu\left(E-E_{0}\right)$, is absolutely continuous with respect to $\nu$.

A measure $\mu$ is semi-convex if every measurable set $E$ contains a measurable set $F$ for which $\mu(F)=\mu(E) / 2$. For any measure $\mu$ and measurable set $E$ let $K(\mu, E)$ be the class of all real-valued measurable functions $\phi$ on $E$ for which $0 \leqq \phi(x)<1$ and $\mu(\{x: \phi(x)<\lambda\})$ $=\lambda \mu(E)$ for $0 \leqq \lambda \leqq 1$. A measure $\mu$ is convex if for every measurable set $E$ the class $K(\mu, E)$ is not empty.

LEMMA 1. If $\mu$ is a semi-convex measure and $E$ is any measurable set then there exists a sequence $\left\{E_{n}\right\}$ of measurable subsets of $E$ such that for every $k=1,2, \cdots$ and any $k$ distinct positive integers $n_{1}, \cdots, n_{k}$

$$
\mu\left(E_{n_{1}} \cap \cdots \cap E_{n_{k}}\right)=\frac{1}{2^{k}} \mu(E) .
$$

Proof. Let $E_{1} \subset E$ be any measurable set with $\mu\left(E_{1}\right)=\mu(E) / 2$. Suppose that measurable sets $E_{1}, \cdots, E_{n}$ have already been constructed so that (1) holds for any $k$ distinct integers $n_{1}, \cdots, n_{k}$, $1 \leqq n_{i} \leqq n, i=1, \cdots, k$. Consider each of the $2^{n}$ sets of the form

$$
E\left(\epsilon_{1}, \cdots, \epsilon_{n}\right)=E_{1}^{\epsilon_{1}} \cap \cdots \cap E_{n}^{\epsilon_{n}}
$$

where $\epsilon_{i}=0$ or $1(i=1, \cdots, n)$ and, for any set $F, F^{1}=F$ and $F^{0}=X$ $-F$. The value of $\mu$ for each such set is $\mu(E) / 2^{n}$. From each set of the form (2) select a measurable subset $F\left(\epsilon_{1}, \cdots, \epsilon_{n}\right)$ so that $\mu\left(F\left(\epsilon_{1}, \cdots, \epsilon_{n}\right)\right)=\mu\left(E\left(\epsilon_{1}, \cdots, \epsilon_{n}\right)\right) / 2$; write $E_{n+1}$ for the union of the selected subsets. The inductive procedure so described proves 
Lemma 1.

LEMMa 2. A semi-convex measure is convex.

Proof. There is no loss of generality in considering non-negative measures only. Let $\mu$ be a non-negative semi-convex measure, let $E$ be any measurable set, and let $\left\{E_{n}\right\}$ be a sequence of sets with the property described in Lemma 1 . If

$$
x \in E_{*}=\lim _{n \rightarrow \infty} \inf E_{n}=\bigcup_{k=1}^{\infty} \bigcap_{n=k}^{\infty} E_{n}
$$

then write $\phi(x)=0$; otherwise write $\phi(x)=\sum_{n=1}^{\infty} \epsilon_{n}(x) / 2^{n}$, where $\epsilon_{n}$ is the characteristic function of $E_{n}$. Clearly $\phi$ is measurable and $0 \leqq \phi(x)<1$. For any dyadically rational number $\lambda=k / 2^{n}$, with $n=0,1,2, \cdots$ and $k=1, \cdots, 2^{n}$, it is easy to verify that

$$
\{x: \phi(x)<\lambda\}=E_{*} \cup\left\{x: \sum_{i=1}^{n} \epsilon_{i}(x) / 2^{i}<\lambda\right\} .
$$

Since the set $\left\{x: \sum_{i=1}^{n} \epsilon_{i}(x) / 2^{i}<\lambda\right\}$ is the union of exactly $k$ pairwise disjoint sets of the form $E_{1}^{\epsilon_{1}} \cap \cdots \cap E_{n}^{\epsilon_{n}}\left(\epsilon_{i}=0\right.$ or $\left.1, i=1, \cdots, n\right)$ and since $\mu\left(E_{*}\right)=0$, it follows that

$$
\mu(\{x: \phi(x)<\lambda\})=\lambda \mu(E) .
$$

The extension of (3) to values of $\lambda$ which are not dyadically rational is an immediate consequence of the countable additivity of $\mu$.

LEMMA 3. Let $\mu$ be a non-negative convex measure and let $E$ be any measurable set. If $\phi \in K(\mu, E)$ and if $\nu$ is a measure absolutely continuous with respect to $\mu$ then $\nu(\{x: \phi(x)<\lambda\})$ is a continuous function of $\lambda, 0 \leqq \lambda \leqq 1$.

Proof. For any $\epsilon>0$ select a $\delta>0$ so that $|\nu(F)| \leqq\left|\nu^{*}(F)\right|<\epsilon$ for every measurable set $F$ for which $|\mu(F)|=\left|\mu^{*}(F)\right|<\delta$. If $0 \leqq \lambda_{1}$ $<\lambda_{2} \leqq 1$ and $\left(\lambda_{2}-\lambda_{1}\right)|\mu(E)|<\delta$ then

$$
\left|\mu\left(\left\{x: \lambda_{1} \leqq \phi(x)<\lambda_{2}\right\}\right)\right|=\left(\lambda_{2}-\lambda_{1}\right)|\mu(E)|<\delta
$$

and therefore $\left|\nu\left(\left\{x: \phi(x)<\lambda_{2}\right\}\right)-\nu\left(\left\{x: \phi(x)<\lambda_{1}\right\}\right)\right|<\epsilon$.

LEMMA 4. If $\mu$ is a convex measure and $E$ and $F$ are any two measurable sets then for every $\lambda, 0 \leqq \lambda \leqq 1$, there exists a measurable set $C(\lambda)$ with the following properties.

(i) $C(0)=E$ and $C(1)=F$.

(ii) $\mu(C(\lambda))=(1-\lambda) \mu(E)+\lambda \mu(F)$. 
(iii) If $\mu$ is also non-negative and if $\nu$ is absolutely continuous with respect to $\mu$ then $\nu(C(\lambda))$ is continuous function of $\lambda$.

COROLLARY. The range of a convex measure is convex.

Proof. Let $\phi$ and $\psi$ be functions in $K(\mu, E-F)$ and $K(\mu, F-E)$ respectively; write $C(\lambda)=(E \cap F) \cup\{x: \phi(x)<1-\lambda\} \cup\{x: \psi(x)<\lambda\}$. The relations (i) are an immediate consequence of the definition of $C(\lambda)$ and (iii) follows from Lemma 3 . The relation (ii) is proved by the following chain of equations.

$$
\begin{aligned}
\mu(C(\lambda)) & =\mu(E \cap F)+\mu(\{x: \phi(x)<1-\lambda\})+\mu(\{x: \psi(x)<\lambda\}) \\
& =\mu(E \cap F)+(1-\lambda) \mu(E-F)+\lambda \mu(F-E) \\
& =(1-\lambda)[\mu(E \cap F)+\mu(E-F)]+\lambda[\mu(E \cap F)+\mu(F-E)] .
\end{aligned}
$$

LEMMA 5. If $\mu=\left(\mu_{1}, \cdots, \mu_{N}\right)$ is non-negative and purely non-atomic and if each $\mu_{i}(1<i \leqq N)$ is absolutely continuous with respect to its predecessor then $\mu$ is convex.

Proof. In view of Lemma 2 it is sufficient to prove that $\mu$ is semiconvex. For $N=1$ this is clear. ${ }^{5}$ Suppose now (in case $N>1$ ) that the $(N-1)$-dimensional measure $\mu^{\prime}=\left(\mu_{1}, \cdots, \mu_{N-1}\right)$ is convex; it follows from the hypotheses that the (one-dimensional) measure $\nu^{\prime}=\mu_{N}$ is absolutely continuous with respect to it. Any measurable set $E$ contains a measurable set $E_{0}$ such that $\mu^{\prime}\left(E_{0}\right)=\mu^{\prime}(E) / 2$; write $F_{0}=E$ $-E_{0}$. If $\nu^{\prime}\left(E_{0}\right)=\nu^{\prime}(E) / 2$ there is nothing to prove; in the contrary case it may be assumed that $\nu^{\prime}\left(E_{0}\right)\left\langle\nu^{\prime}(E) / 2\right.$ and $\left.\nu^{\prime}\left(F_{0}\right)\right\rangle \nu^{\prime}(E) / 2$. Lemma 4 applied to $\mu^{\prime}, \nu^{\prime}, E_{0}, F_{0}$ in place of $\mu, \nu, E, F$ yields the existence of a number $\lambda, 0 \leqq \lambda \leqq 1$, such that $\nu^{\prime}(C(\lambda))=\nu^{\prime}(E) / 2$ and

$$
\begin{aligned}
\mu^{\prime}(C(\lambda)) & =(1-\lambda) \mu^{\prime}\left(E_{0}\right)+\lambda \mu^{\prime}\left(F_{0}\right) \\
& =(1-\lambda) \frac{\mu^{\prime}(E)}{2}+\lambda \frac{\mu^{\prime}(E)}{2}=\frac{\mu^{\prime}(E)}{2} .
\end{aligned}
$$

It follows that $\mu(C(\lambda))=\mu(E) / 2$, and consequently the inductive proof of Lemma 5 is complete.

LEMMA 6. A purely non-atomic non-negative measure is convex.

Proof. If $\mu=\left(\mu_{1}, \cdots, \mu_{N}\right)$ is purely non-atomic, write $\mu_{i}^{\prime}=\sum_{n=1}^{N} \mu_{n}$. Since $\mu^{\prime}=\left(\mu_{1}^{\prime}, \cdots, \mu_{N}^{\prime}\right)$ satisfies the conditions of Lemma $5, \mu^{\prime}$ is convex. Let $T$ be the inverse of the linear transformation which carried $\mu$ into $\mu^{\prime}, \mu=T \mu^{\prime}$. From the linearity of $T$ and the semi-convexity of $\mu^{\prime}$ it follows that $\mu$ is semi-convex and hence convex.

${ }^{5}$ Cf. Lemma 2 of the paper cited in footnote 2. 
LeMma 7. A purely non-atomic measure is convex.

Proof. For each $i=1, \cdots, N$ there exists a measurable set $E_{i}$ such that $\mu_{i}\left(E \cap E_{i}\right) \geqq 0$ and $\mu_{i}\left(E-E_{i}\right) \leqq 0$ for every measurable set $E$. On all measurable subsets of any one of the $2^{N}$ sets of the form $E_{1}^{\epsilon_{1}} \cap \cdots \cap E_{N}^{\epsilon_{N}}\left(\epsilon_{i}=0\right.$ or $\left.1, i=1, \cdots, N\right)$ each of the measures $\mu_{1}, \cdots, \mu_{N}$ is of constant sign. Lemma 6 may then be applied to the set function $\mu_{\epsilon_{1}}, \cdots, e_{N}=\left( \pm \mu_{1}, \cdots, \pm \mu_{N}\right)$, where the ambiguous sign is chosen in each case so that $\mu_{\epsilon_{1}}, \ldots, \epsilon_{N}$ is a non-negative measure. The convexity of $\mu_{\epsilon_{1}}, \ldots, e_{N}$ and the disjointness of any two distinct ones of the sets $E_{1}^{\epsilon_{1}} \cap \cdots \cap_{N}^{\epsilon_{N}}$ imply the convexity of $\mu$.

LEMMA 8. If $\mu$ is a measure with convex range $R$ and $\Pi$ is a supporting plane of the closure $\bar{R}$ then $R \cap \Pi \neq 0$.

Proof. Let $L$ be a real linear function on the value space of $\mu$ such that $\Pi=\{\xi: L(\xi)=\lambda\}$ for some real number $\lambda$. The fact that $\Pi$ supports $\bar{R}$ can be expressed (after a suitable choice of signs) by the relation $\lambda=\inf \{L(\mu(E)): E \in S\}$. Since a numerical measure always assumes its minimum there exists a measurable set $E$ for which $L(\mu(E))=\lambda$; for this $E$, clearly, $\mu(E) \in R \cap \Pi .^{\circ}$

LEMMA 9. If $\mu$ is a measure with range $R$ and $\xi \in R$ then there exists a measure $\mu^{\prime}$ whose range $R^{\prime}$ is obtained from $R$ by a translation by $-\xi$; if $\mu$ is convex then $\mu^{\prime}$ is also.

Proof. If $\xi=\mu(A)$, write $\mu^{\prime}(E)=\mu(E-A)-\mu(E \cap A)$. A trivial calculation shows that for every measurable set $E, \mu^{\prime}(E)=\mu((E-A)$ $\cup(A-E))-\xi \quad$ (so that $\left.R^{\prime} \subset R-\xi\right)$, and $\mu(E)-\xi=\mu^{\prime}((E-A)$ $\cup(A-E)$ ) (so that $R-\xi \subset R^{\prime}$ ). If $\mu$ is convex and $E$ is any measurable set, let $F_{-}$and $F_{+}$be measurable subsets of $E-A$ and $E \cap A$ respectively such that $\mu\left(F_{-}\right)=\mu(E-A) / 2$ and $\mu\left(F_{+}\right)=\mu(E \cap A) / 2$. The equation $\mu^{\prime}\left(F_{-} \cup F_{+}\right)=\mu^{\prime}(E) / 2$ implies that $\mu^{\prime}$ is serni-convex and hence convex.

\section{LEMMA 10. The range of a convex measure is closed.}

Proof. Let $\mu=\left(\mu_{1}, \cdots, \mu_{N}\right)$ be a convex measure with range $R$. If $R$ is one-dimensional then $\mu(E)=\mu_{0}(E) \xi$, where $\mu_{0}$ is a numerical measure and $\xi$ is a vector, and the result is clear. Assume next, inductively, that $N>1$ and that the result is known whenever the dimension of $R$ is not greater than $N-1$. The proof of closure will be carried out by showing that $\bar{R} \cap \Pi \subset R$ for every supporting plane II of $\bar{R}$.

- This lemma and its proof are given in Liapounoff's first paper, cited in footnote 1. 
Since, by Lemma $8, \Pi$ meets $R$ in at least one point $\xi$, and since, by Lemma $9, R-\xi$ is also the range of a convex measure (and is supported at the origin by $\Pi-\xi$ ), there is no loss of generality in assuming that $\Pi$ contains the origin. Let $L$ be a linear function such that $\Pi=\{\xi: L(\xi)=0\}$ and $\inf \{L(\mu(E)): E \in S\}=0$; then the function $\nu$, defined by $\nu(E)=L(\mu(E))$, is a non-negative numerical measure. Let $E_{0}$ be a measurable set such that $\nu\left(E_{0}\right)=0$ and that the measure $\mu^{-}$, defined by $\mu^{-}(E)=\mu\left(E-E_{0}\right)$, is absolutely continuous with respect to $\nu$. Let $R^{+}$be the range of the measure $\mu^{+}$, defined by $\mu^{+}(E)=\mu\left(E \cap E_{0}\right)$. Since $L\left(\mu^{+}(E)\right)=L\left(\mu\left(E \cap E_{0}\right)\right)=\nu\left(E \cap E_{0}\right)=0$, $R^{+} \subset \Pi$, and therefore, by the induction hypothesis, $R^{+}$is closed.

If $\xi \in \bar{R} \cap \Pi$ then there exists a sequence $\left\{E_{n}\right\}$ of measurable sets such that $\mu\left(E_{n}\right) \rightarrow \xi$ and therefore $\nu\left(E_{n}\right)=L\left(\mu\left(E_{n}\right)\right) \rightarrow L(\xi)=0$. It follows that $\mu\left(E_{n}-E_{0}\right)=\mu^{-}\left(E_{n}\right) \rightarrow 0$ and therefore that $\mu^{+}\left(E_{n}\right)$ $=\mu\left(E_{n} \cap E_{0}\right) \rightarrow \xi$. Since $\mu^{+}\left(E_{n}\right) \in R^{+}$and $R^{+}$is closed it follows, finally, that $\xi \in R^{+} \subset R$, and therefore that $\bar{R} \cap \Pi \subset R$.

LEMMA 11. The range of a non-negative measure is closed.

Proor. If $\mu=\left(\mu_{1}, \cdots, \mu_{N}\right)$ is a non-negative measure let $T=\left(t_{i j}\right)$ be a nonsingular $N \times N$ matrix with positive elements and write $\mu^{\prime}=\left(\mu_{1}^{\prime}, \cdots, \mu_{N}^{\prime}\right)=T \mu$. Each of the non-negative measures $\mu_{1}^{\prime}, \cdots, \mu_{N}^{\prime}$ is absolutely continuous with respect to every other one. Since the range of $\mu$ is obtained from this range of $\mu^{\prime}$ by applying $T^{-1}$ it may be assumed that $\mu$ itself has the absolute continuity property. It follows that any atom of each coordinate is an atom of all others. If $Y$ is the union of the (at most countably many) common atoms and $Z=X-Y$, and if $\mu^{\prime}(E)=\mu(E \cap Y)$ and $\mu^{\prime \prime}(E)=\mu(E \cap Z)$ then $\mu^{\prime}$ is purely atomic and $\mu^{\prime \prime}$ is purely non-atomic. The range $R$ of $\mu$ is the vector sum of the respective ranges $R^{\prime}$ and $R^{\prime \prime}$ of $\mu^{\prime}$ and $\mu^{\prime \prime}$. The range $R^{\prime \prime}$ is closed by Lemmas 7 and 10; the proof of Lemma 3 of my preceding paper (cited in footnote 3 ) applies without any change to prove that $R^{\prime}$ is closed. Since the vector sum of two compact sets is compact the proof of Lemma 11 is complete.

The proof of Lemma 7 shows that the range of an arbitrary (not necessarily non-negative) $N$-dimensional measure is the vector sum of $2^{N}$ sets each of which may be shown to be closed by Lemma 11. The preceding results may, accordingly, be summed up as follows.

THEOREM. The range of every measure is closed; the range of every purely non-atomic measure is convex.

University of Chicago 